\title{
Lack of Consensus and Dense Content in Informational Letters on Benign Gynecological Surgery
}

\author{
Sofia Widetun* ${ }^{*}$, Margareta Nilsson, Mats Löfgren, Maria Lindqvist \\ Department of Clinical Sciences, Obstetrics and Gynecology, Umeå University, Umeå, Sweden \\ Email: *sofia.widetun@ptj.se
}

How to cite this paper: Widetun, S., Nilsson, M., Löfgren, M. and Lindqvist, $M$. (2018) Lack of Consensus and Dense Content in Informational Letters on Benign Gynecological Surgery. Open Journal of Obstetrics and Gynecology, 8, 1583-1595. https://doi.org/10.4236/ojog.2018.814159

Received: November 13, 2018

Accepted: December 25, 2018

Published: December 28, 2018

Copyright (c) 2018 by authors and Scientific Research Publishing Inc. This work is licensed under the Creative Commons Attribution International License (CC BY 4.0).

http://creativecommons.org/licenses/by/4.0/

\begin{abstract}
Objective: This study maps the contents of departmental informational letters and explores whether the national GynOp letters live up to the patients' expectations and needs. Introduction: Patients who are well informed before undergoing surgery experience reduced stress and increased understanding of the postoperative process. Although providing patients with written information before gynecological surgery is widely used and assumed important, no study has investigated what information patients truly need. Methods: In 2014, all 59 gynecological departments in Sweden were asked to provide the information letter they send to patients before hysterectomy on benign indication. 32 letters were analyzed using frequency analysis. In addition, three focus groups were conducted and these data were submitted to Qualitative Content Analysis. Results: The analysis of the information letters showed great variation and discrepancy in pre-operative information. The analysis of the focus groups resulted in the theme You can't see the forest for the trees, reflecting that, the women found it very difficult to identify the most important information among the massive amount information received. Conclusions: The informational letters did not meet the patients' expectations and needs. Practice implications: This study could serve as a foundation for the content of informational letters.
\end{abstract}

\section{Keywords}

Patient Information, Gynecological Surgery, Quality Register, Qualitative, Focus Group Discussions

\section{Introduction}

Patients who are well informed before undergoing surgery experience reduced 
stress [1], increased understanding of the postoperative process [2], and increased participation in decision-making [3]. A combination of written and oral information is preferred by the patients [4]. Furthermore, written, structured, and comprehensive information is perceived to be valuable [5]. Preoperative informational letters are often provided to help patients understand their surgery and recovery. For example, Swedish gynecological departments provide such a letter to patients before gynecological surgery. Although these locally-distributed letters explain local routines, surgical methods, and possible complications, they rarely consider the patient's perspective or needs.

The Swedish National Quality Register of Gynecological Surgery (GynOp), formed in 1997, aims to improve gynecological healthcare. The register includes one preoperative and two postoperative questionnaires together with records from the surgery contributed by the operating surgeon [6]. The GynOp preoperative questionnaires include informational letters about the upcoming surgery. Although the participating departments are supposed to modify the letters so it reflects their local routines, the letters are rarely revised. Often the original GynOp letter is distributed as a supplement to departmental information, meaning that the patients receive a vast amount of information at once. The GynOp informational letter about hysterectomy is 493 words long, the letter about incontinence surgery 420 words and the letter about prolapse surgery is 1050 words, not including local information.

Per current Swedish legislation, patients are entitled to receive full information about their healthcare [7]. Although the local and national informational letters are intended to satisfy this legal obligation, to our knowledge no study has examined what kind of information patients desire.

This study has two aims: i) to map the contents of the local informational letters and identify the differences between departments and ii) to explore whether GynOp's national informational letters meet the expectations and needs of the patients.

\section{Material and Methods}

\subsection{Local Informational Letters}

All 59 gynecological departments in Sweden were asked to submit their local informational letters concerning hysterectomy. Of these, 32 responded (Figure 1). The letters were screened and the data were analyzed using simple frequency analysis (SPSS 21.0). Information on local affiliation such as routines for admission and wound dressing were excluded from the material.

\subsection{Focus Group Discussion}

Focus group discussions (FGD) are a qualitative method where the participants share their experiences in a group. To explore patients' experiences with the GynOp letters, three FGD were conducted. The participants were divided into different types of surgery-hysterectomy, incontinence, or prolapse surgery. The selection consisted of women who had undergone surgery during the previous 


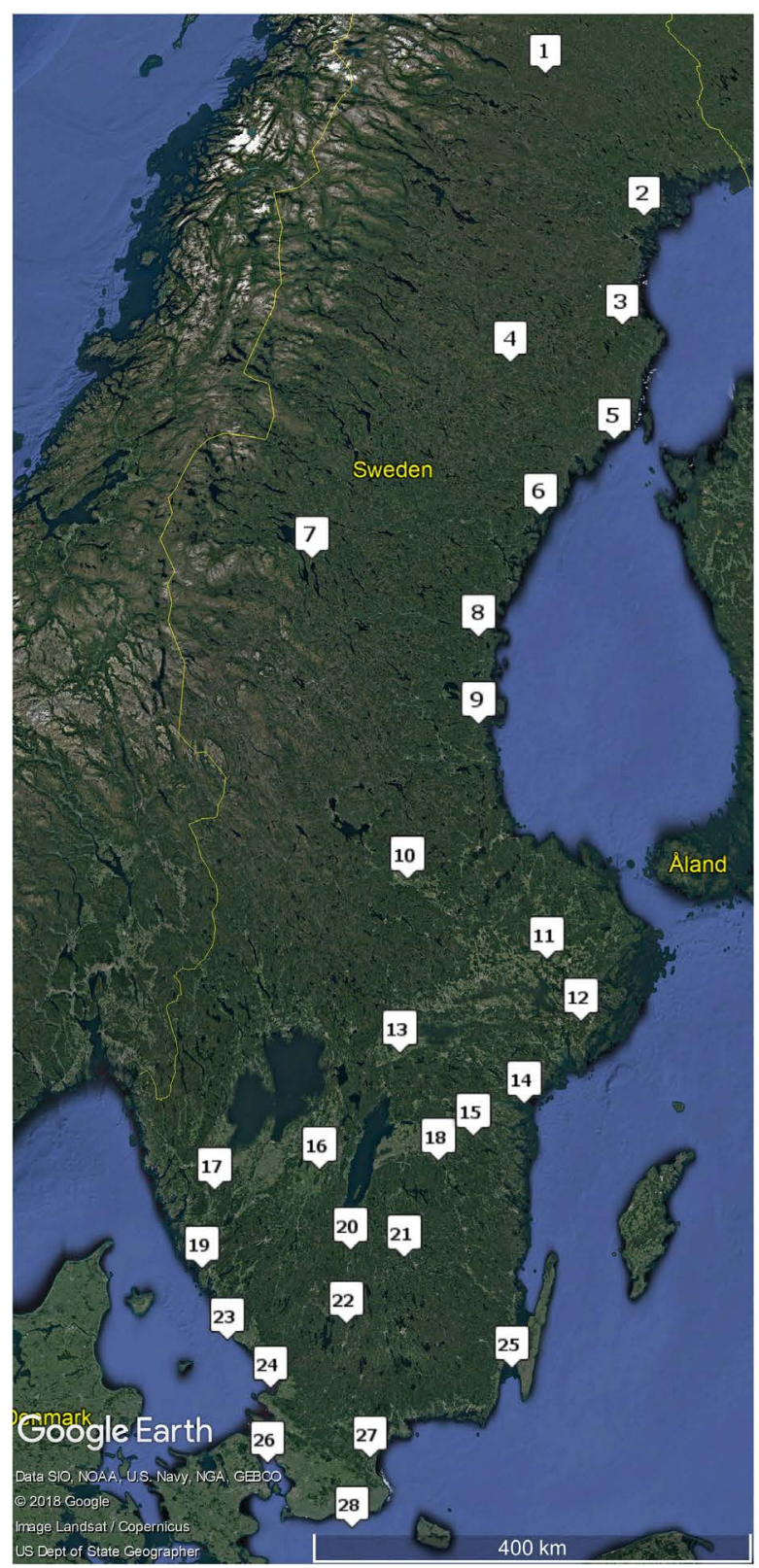

1. Gällivare Hospital.

2. Sunderby Hospital.

3. Skellefteå Hospital.

4. Lycksele Hospital.

5. Norrland University Hospital.

6. Örnsköldsvik Hospital.

7. Östersund Hospital.

8. Sundsvall Hospital.

9. Hudiksvall Hospital.

10. Falu Hospital.

11. Uppsala University Hospital.

12. Karolinska Institutet, Aleris Specialist Care, Cevita Care.

13. Örebro University Hospital.

14. Nyköping Hospital.

15. Vrinnevi Hospital.

16. Skaraborg Hospital.

17. Norra Älvsborg County Hospit-al.

18. Linköping University Hospital.

19. Sahlgrenska Hospital, Carlanderska Hospital, Östra Hospital.

20. Ryhov County Hospital.

21. Höglands Hospital.

22. Värnamo Hospital.

23. Halland Hospital of Varberg.

24. Halland Hospital of Halmstad.

25. Kalmar County Hospital.

26. Helsingborg Hospital.

27. Kristianstad Central Hospital.

28. Ystad Hospital.

Figure 1. Included departments that contributed with their informational letter.

12 - 24 months, hysterectomy in Gothenburg, incontinence surgery in Kungsbacka or prolapse surgery in Umea, and who had answered all the GynOp questionnaires. These women were invited to participate via telephone and were contacted in order of most recent surgery and with as great an age diversity as possible. The goal was to create three groups of seven (Figure 2). Women aged 80 years or older were excluded. In the end, the three groups consisted of four, five, and six women. The women were informed orally about their right to end their participation at any time. Before the FGD were performed, the women received the letters from GynOp over again. The interviewer used a guide with four subject areas: experiences and attitudes towards GynOp, reactions to the informational letters, reactions to the questions in the questionnaires, and future 
development and adaption of the registry. This study presents the reactions to the informational letters. To achieve a good atmosphere for these discussions, the FGD were performed in private rooms in the hospitals where the participants had undergone surgery (Sahlgrenska Hospital, The Hospital of Halland Kungsbacka, and Norrland University Hospital) [8]. The FGD lasted about one and a half hours under the overview of second author and a moderator chosen by SALAR (Swedish Association of Local Authorities and Regions).

The FGD were digitally recorded and transcribed by a secretary. The material was listened and read through thoroughly by the first, second and fourth author before analysis. The discussions were analyzed using Qualitative Content Analysis (QCA) to explore how the participants experienced the letters and how they preferred them to be presented [9] [10]. Meaning units, condensed meaning units and codes were identified (Table 1). Then sub-categories, and categories where formed and a theme was identified [10]. For the purpose of trustworthiness, the initial analysis was conducted by SW, MN, and ML. The sub-categories, categories, and the emerging theme were negotiated and finally agreed on by all three researchers.

\subsection{Ethical Approval}

The study was approved by the Ethical Review Board, Umeå University, Sweden

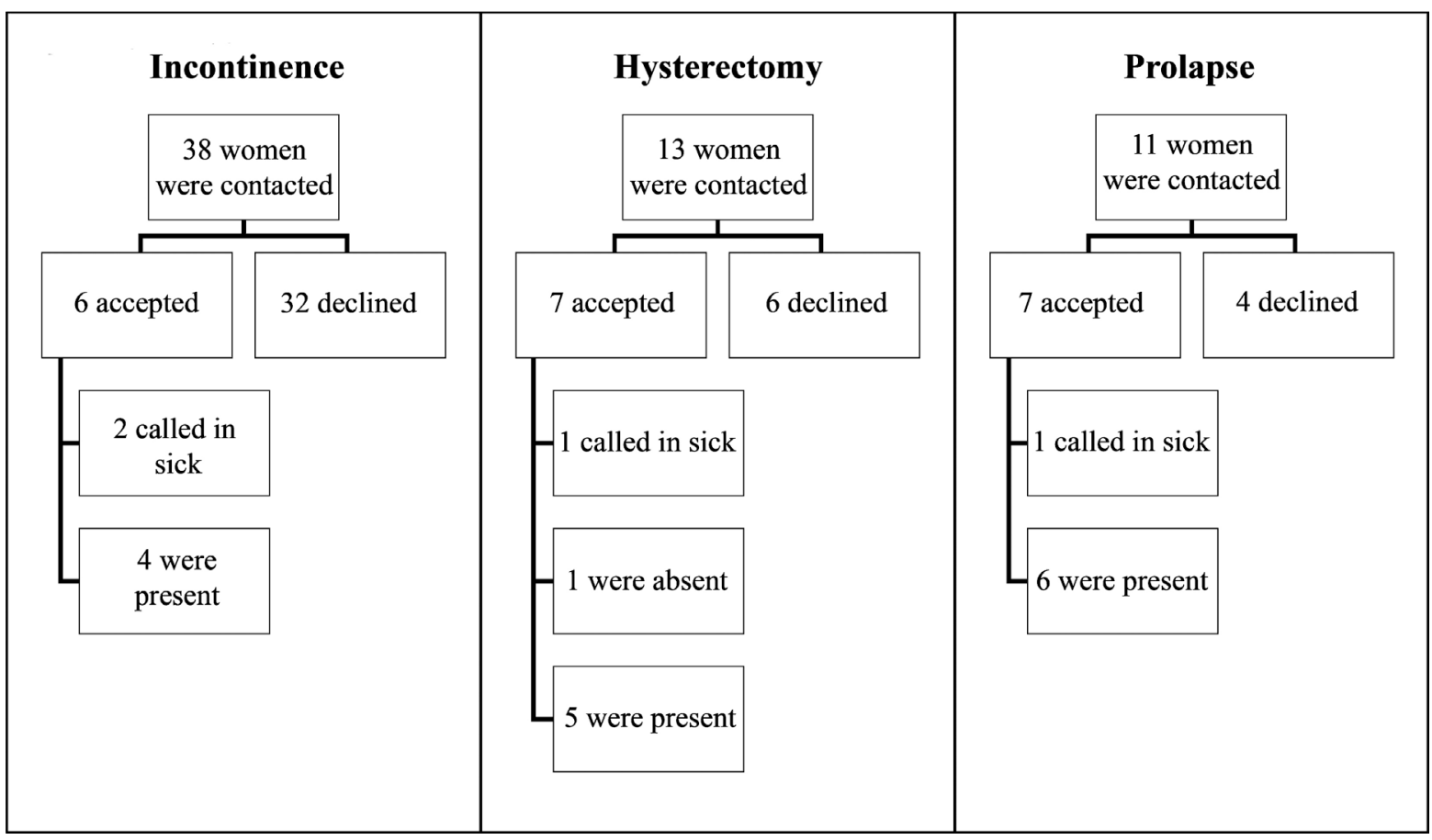

Figure 2. Participant selection to focus groups; women who had undergone incontinence, hysterectomy or prolapse surgery participated in separate groups.

Table 1. Example of text condensation.

\begin{tabular}{ccc}
\hline Meaning unit & Condensed meaning unit & Code \\
\hline $\begin{array}{c}\text { "It feels really good if you're able to help others ... to be able to help so that, well, you } \\
\text { can process all the answers and then it will be helpful for them either in ..." }\end{array}$ & It feels good to help others. & Help others \\
\hline
\end{tabular}


(Dno: 2013-401-32M; Supplement to 08-076M). Collection and management of patient data in health systems and health registers are regulated through the Swedish Patient Law. Participation in GynOp is voluntary.

\section{Results}

\subsection{Local Informational Letters}

Common causes for hysterectomy on benign indication were described in $43.8 \%$ $(n=14)$ of the local informational letters. A large percentage of the informational letters, $87.5 \%(n=28)$, provided information about different surgical methods such as abdominal, vaginal, or laparoscopic hysterectomy and 62.5\% $(\mathrm{n}=20)$ described the difference between total and subtotal hysterectomy. Only 25\% ( $\mathrm{n}=$ 8) of the informational letters provided information about the necessity of Pap smears after subtotal hysterectomy (Table 2).

The vast majority of the informational letters described sick leave and the duration of hospital stay, how long a patient should wait before lifting heavy objects or engaging in coitus. $81.3 \%(n=26)$ of the letters instructed the women to avoid heavy lifting and other strenuous exercises and $84.4 \%(\mathrm{n}=27)$ instructed the women to abstain from intercourse. However, the letters did not provide the same information regarding time span (Table 3 ).

Sometimes the patients' ovaries are removed during a hysterectomy, leading to menopause. This information was found in $84.4 \%(n=27)$ of the letters. Information regarding how to avoid postoperative complications varied: $59.4 \%$ (n $=19)$ of the letters provided advice against bathing and $40.6 \%(n=13)$ of the letters advised against the use of tampons.

The importance of seeking healthcare in case of serious complications or alarming symptoms to prevent permanent or even life threatening conditions were found in $65.6 \%(n=21)$ of the letters. These letters also provided proper contact information. Strikingly, only $6.3 \%(n=2)$ of the letters provided information about the benefits of the operation.

\subsection{Focus Group Discussions}

The focus group analysis resulted in one overarching theme: You can't see the forest for the trees. This theme reflects the participants' desire to be given simple and accessible information but in reality being overburdened with dense and unnecessary information. That is, the participants felt the letters and overall communication from healthcare providers was overly complicated and burdensome. Although they were eager to learn more about the surgery, they found the written explanations too difficult. They felt they were being given too much and the wrong type of information. This overload of information was counterproductive as the most important information was lost in the details.

During the analysis three main categories were identified: Experiencing dense contents, Experiencing confusion, and Feelings of being prepared (Table 4). 
Table 2. Contents of the local informational letters.

Information about the preoperative course and how the operation is performed

Explanation of different surgical methods ${ }^{\mathrm{a}}$

The signification of total or subtotal hysterectomy ${ }^{a}$

Common causes of hysterectomy $\mathrm{y}^{\mathrm{a}}$

Normal time span of surgery

Discontinuation of specific drugs before surgery ${ }^{\mathrm{b}}$

$9(28.1)$

Information about the postoperative course

Normal time span of sick leave ${ }^{\mathrm{a}}$

$30(93.8)$

Normal time span of hospital stay ${ }^{\mathrm{a}}$

$29(90.4)$

Menopausal symptoms after removal of ovaries ${ }^{\mathrm{a}}$

Avoidance of coitus $^{c}$

$27(84.4)$

Avoidance of heavy lifting ${ }^{c}$

$26(81.3)$

Common postoperative symptoms $s^{\mathrm{a}}$

$25(78.1)$

Analgesic regime after discharge $\mathrm{e}^{\mathrm{a}}$

$24(75.0)$

Removal of stitches or clips ${ }^{\mathrm{b}}$

$23(71.9)$

Exhortation to seek medical help if alarming symptoms arise ${ }^{\mathrm{b}}$

$21(65.6)$

Routines for returning visit ${ }^{\mathrm{a}}$

Avoidance of bathing

Avoidance of using tampons ${ }^{c}$

$13(40.6)$

Menstruation and pregnancy after hysterectomy ${ }^{a}$

$13(40.6)$

Routines of notifying histopathological results ${ }^{\mathrm{b}}$

The necessity of future Pap smears ${ }^{\mathrm{a}}$

Possible improvements to be expected ${ }^{\mathrm{a}}$

$2(6.3)$

a. Supported by statistics from the GynOp register or other evidence-based source. b. Without scientific proof. c. Individual routines.

Table 3. Recommended time spans after surgery.

\begin{tabular}{|c|c|c|c|c|c|}
\hline \multirow{2}{*}{ Time span of hospital stay } & 1 - 2 days & 3 days & $4-5$ days & No set time & Missing data \\
\hline & $20(62.5)$ & $6(18.8)$ & $2(6.3)$ & $1(3.1)$ & $3(9.4)$ \\
\hline \multirow{2}{*}{ Time span of sick leave } & 1 week & 2 - 4 weeks & $>4$ weeks & No set time & Missing data \\
\hline & $1(3.1)$ & $24(75.0)$ & $3(9.4)$ & $2(6.3)$ & $2(6.3)$ \\
\hline \multirow{2}{*}{ Time span of avoiding coitus } & 2 weeks & 3 weeks & 4 weeks & $>4$ weeks & Missing data \\
\hline & $1(3.1)$ & $6(18.8)$ & $14(43.8)$ & $6(18.8)$ & $5(15.6)$ \\
\hline \multirow{2}{*}{ Time span of avoiding heavy lifting } & 2 - 3 weeks & 4 weeks & $>4$ weeks & No set time & Missing data \\
\hline & $9(28.1)$ & $12(37.5)$ & $1(3.1)$ & $4(12.5)$ & $6(18.8)$ \\
\hline
\end{tabular}

All the values are expressed as number and percentage of departments regarding the variable; $\mathrm{n}(\%)$. 
Table 4. Identified theme, categories and subcategories.

\begin{tabular}{|c|c|c|}
\hline Theme & Category & Subcategory \\
\hline \multirow{9}{*}{$\begin{array}{c}\text { You can't see the forest for } \\
\text { the trees }\end{array}$} & \multirow{3}{*}{ Experiencing dense content } & Dense text \\
\hline & & A lot of text \\
\hline & & Difficult text \\
\hline & \multirow{4}{*}{ Experiencing confusion } & Confusing message \\
\hline & & Lack of information \\
\hline & & Perceived ambiguity \\
\hline & & The time factor \\
\hline & \multirow{2}{*}{ Feelings of being prepared } & Relevant information \\
\hline & & Detailed information \\
\hline
\end{tabular}

\subsubsection{Experiencing Dense Contents}

Many of the participants felt the letters were too long and too dense. When there was too much information presented at once, the participants lost interest and scanned it briefly rather than reading it thoroughly.

"I thought it was so much text, when you are about to go through ... and then you just skimmed it through.”-FGD 2, hysterectomy.

The dense text contributed to the experience of being overwhelmed with information before the surgery. This experience of being overwhelmed was complicated further by the fact that they often received the informational letter at the same time as they received the GynOp letter, information about the GynOp registry, and the questionnaire.

"I remember receiving heaps in that envelope (laugh), it was papers from GynOp, from the ward, and from ..."-FGD 2

The participants also questioned whether people with reading disabilities or who are not fluent in Swedish would be able to understand the information.

"No, it was something I noticed about the text that ... when I read it ... I have dyslexia and I thought the text was difficult."-FGD 2

The participants also felt the text was too homogenous, which reduced the motivation to read it. The participants wanted a straightforward text that presented just enough information to give them an overview of their surgery, prognosis, and recovery. In addition, the participants felt the layout of the letters could be improved. For example, they believed all the information could be presented on one page in distinct paragraphs with the most important information highlighted.

"Well, for example, if you have one part of the text that is presented as a bulleted list and then there's a box and then there's something else to make the paper come alive ... it's the layout that $1 \mathrm{~m}$ after."-FGD 1, incontinence

The participants experienced difficulties digesting all the information, as their thoughts were preoccupied by the upcoming surgery.

\subsubsection{Experiencing Confusion}

The GynOp informational letters sometimes caused confusion, leading to feelings of uncertainty and anxiety. For example, different indications for surgery 
and surgical methods were described in the same letter. The participants found it difficult to understand what information that was relevant to them.

"Also, it's difficult when they write that it all depends on what kind of surgery they decide upon."-FGD 3, prolapse

The participants perceived that some information-such as postoperative symptoms, the risk of a relapse, and routines for revisits-was insufficient.

"No, nothing about maybe waiting a while or the healing process."-FGD 3

Furthermore, anxiety was experienced if no instructions were presented on how to act and who to contact if any problems or questions occurred after surgery.

The participants requested information regarding their surgery earlier in the process. A few weeks before the surgery or at the enrolment were considered too late. In addition, they wanted discussions about risks and the potential improvement to take place before they decided to undergo surgery.

"I also looked online but it would have been great with one site where you could get all the information instead of looking it up on a bunch of different ones, reading a bit here and a bit there ... I felt that I needed to know what was going to happen."-FGD 2

\subsubsection{Feelings of Being Prepared}

The participants highly valued information given before the surgery, eliminating the risk of going through surgery unprepared. The participants' reactions were positive if the information was relevant and described in a straightforward way. Information regarding the postoperative convalescence as well as the potential of improvement or the risk of complications generated a sense of reassurance.

"It felt good that it said something about the time after the surgery ... often you get a piece of paper and some painkillers and then you just stumble out leaning on a family member ... and then it takes at least a couple of days before you read it. But there you got the information beforehand."-FGD 1

Information about the Swedish Personal Data Act was conveyed through links to The Swedish Government and the Data Protection Authority [11] [12]. The participants considered this as an excellent way of getting information. In addition to a more succinct and focused letter, some of the participants wanted access to a webpage that provided statistics regarding complications and results. This strategy would allow patients to delve into information according to their needs and desires, ignoring information they felt was unhelpful or they were unprepared to understand.

"Just like everybody else, refer to a website so you have a website with, well, all that you want ... then you won't need it all in the letter."-FGD 1

\section{Discussion and Conclusion}

\subsection{Discussion}

Information is difficult. Our main findings showed that patients in Sweden receive too much information and not the kind of information desired before undergoing gynecological surgery. This finding stands in great contrast to the in- 
tent of the Swedish Patient Law, which states that patients should receive all available information [7]. Herber et al. show that patient information leaflets about medications with too much risk information conveyed in a formal way provoked negative emotions rather than a sense of security [13]. Patients would like the ability to choose how much information they receive and they would like information to be accessible early on in the process. At about 450 words, informational letters before surgery were considered too long even though legislation and patients require full and adequate information. These conflicting needs create an impossible demand for any informational letter.

Today, the internet makes it possible to customize the amount of information patients' request. Our results showed that women wanted short letters of about 250 words that only included the most relevant information and with links to websites for more information. The solution could be a website with evidence-based information available, a web site that describes pros and cons of the surgery, indications, methods, and preferably the positive effects. In this way, the Swedish Patient Law would be fulfilled without overburdening the patients with information. Patients should be involved in developing such a website. A study about communicating treatment information to cancer patients using a website shows that input from patients results in a more comprehensive and useable website. It is also found that patients desire information that the professionals had not foreseen [14]. However, this method could also be criticized since some patients may not use the internet.

Furthermore, we found that the gynecology departments rarely included evidence-based information in their informational letters. Consequently, the letters included great variations and discrepancies. Evidence-based information related to what women can affect themselves, such as appropriate activity in the postoperative course, is lacking completely. A systematic review carried out in 2013 concludes that there are limited data to guide many aspects of postoperative care after female pelvic surgery, including abstinence from sexual activity, physical activity restrictions, and pelvic muscle exercise [15]. However, a study of 204 women who had undergone hysterectomy shows that preoperative education about potential negative sexual outcomes may enhance satisfaction with the outcomes of hysterectomy, independent of whether negative sexual outcomes were experienced [16]. Further research is urgently needed in order to provide evidence-based information on postoperative factors that women can influence themselves.

Although the GynOp letters refer to evidence-based information, they were too inadequate regarding information to women. Both the local informational letters and the GynOp letters explained different surgical methods. In addition, they differed in describing different surgical indications. For most women, other types of surgery were redundant. The woman is undergoing surgery because of her own physical illness and the method is often already decided upon. Modifying the informational letters by including the local information could be a solu- 
tion. Through this strategy, patients would receive the necessary medical facts and the local routines in one letter. Contradictions regarding information can also arise as the result of internet research uncovering contradictory information.

Only a minority of the gynecological departments in Sweden described the benefits of surgery such as the improvements you can expect and how long it will take to recover. An explanation could be that surgery on benign indication is often performed "solely" to enhance quality of life. Additionally, gynecological surgery treats female disorders, problems that often are described as hidden or forgotten [17]. Another reason could be a lack of knowledge regarding the number of women who experience improvement and how long it takes to improve [6] [18].

It was obvious that the participants in this study wanted to know the pros and cons of going through surgery. To receive information about the risks only a few weeks before the surgery caused anxiety and worry. The women wanted the information much earlier in the process and they wanted this information to always be available, for example, via the internet. Similarly, Wade et al. concluded that women want accurate and useful information at an appropriate time before a hysterectomy [19].

Several departments urge patients to seek healthcare if alarming symptoms occur during the initial healing process. However, information explaining when the surgery is considered successful and what the acceptable complications are without taking further actions is omitted. This omission causes anxiety that could affect the postoperative course. In a study of women undergoing hysterectomy on benign indication, a relationship between high levels of preoperative anxiety and high levels of postoperative pain is found [20]. However, a qualitative study shows that pre-operative education about post-operative precautions before hip replacement surgery help patients feel less anxious [21]. Hence, one can hypothesize that sufficient information before gynecological surgery could reduce preoperative anxiety and postoperative pain.

$54 \%$ of the contacted departments contributed with their requested local informational letters, a percentage that represents a majority of the Swedish departments and strengthens the representativeness of the results. However, a higher response rate would probably lead to even greater variability.

The focus groups consisted of 15 women in total, and we believe the participants represent a significant variation of experiences including age, ethnicity, place of residence, and hospital performing the surgery. There may be some selection bias associated with this study, but it is unclear whether the women who were satisfied or the women who experienced problems with their surgery would have been more likely to participate. However, due to the variation in the findings, the authors believe that the composition of the FGD were satisfying. Furthermore, the moderator may have influenced the content of the discussions although an interview guide was used.

In summary, patients' wishes and opinions have never been taken into con- 
sideration while creating either the GynOp letters or the local informational letters. This lack of awareness probably explains why none of the letters met the women's expectations. Regardless of geographical location, before undergoing gynecological surgery women in Sweden should be well informed and receive equal evidence-based information written from a patient's perspective.

\subsection{Conclusions}

This study demonstrates the complexity of informing patients in a way that is understandable and fulfils the needs and desires of patients. It seems that patients wanted only the most relevant information in an informational letter; the letters should include the most serious and the most common complications as well as the positive effects of the surgery and nothing more. To compose such a letter, which should be shorter than 450 words and still meet the criteria, is a challenge. The writers' suggestion is that departments and other institutions responsible for producing patient information should include patients during the process, in that way securing that the information is relevant and wanted. More information, including evidence-based information, statistical information, and postoperative routines, could be presented via a website for those individuals who want to know more. Information about the website should be included in the letter.

In conclusion, informational letters distributed before benign gynecological surgery do not meet the patient's needs or expectations. However, we have great trust in future letters to be written in a much more patient centered mindset and with the patients input included, thereby fulfilling wishes and needs of the patients. We believe this study could serve as the foundation for developing more effective (that is, more patient-centered) informational letters.

\section{Acknowledgements}

We would like to express our sincere thanks to the participating women in this study for sharing their valuable experiences and their time. Furthermore, we thank the Swedish National Quality Registry of Gynecological Surgery (GynOp) as well as the Swedish Government and the Swedish Association of Local Authorities and Regions, which both support GynOp.

\section{Funding}

No financial support was received for this study.

\section{Informed Consent and Patient Details}

We confirm all personal identifiers have been removed or disguised so the persons described are not identifiable and cannot be identified through the details of the story.

\section{Previous Presentations}

This study has been presented (oral presentation and poster) at the yearly con- 
ference of SFOG (Swedish Society of Obstetrics and Gynecology) in $25^{\text {th }}$ of August 2015, Jonkoping, Sweden.

\section{Conflicts of Interest}

The authors declare no conflicts of interest regarding the publication of this paper.

\section{References}

[1] Ayyadhah Alanazi, A. (2014) Reducing Anxiety in Preoperative Patients: A Systematic Review. British Journal of Nursing, 23, 387-393.

https://doi.org/10.12968/bjon.2014.23.7.387

[2] O’Brien, L., McKeough, C. and Abbasi, R. (2013) Pre-Surgery Education for Elective Cardiac Surgery Patients: A Survey from the Patient's Perspective. Australian Occupational Therapy Journal, 60, 404-409. https://doi.org/10.1111/1440-1630.12068

[3] Vuorma, S., Rissanen, P., Aalto, A.M., Hurskainen, R., Kujansuu, E. and Teperi, J. (2003) Impact of Patient Information Booklet on Treatment Decision: A Randomized Trial among Women with Heavy Menstruation. Health Expectations, 6, 290-297. https://doi.org/10.1046/j.1369-7625.2003.00225.x

[4] Merle, V., Marini, H., Rongère, J., Tavolacci, M.P., Scotté, M. and Czernichow, P. (2011) Does an Information Leaflet about Surgical Site Infection (SSI) Improve Recollection of Information and Satisfaction of Patients? A Randomized Trial in Patients Scheduled for Digestive Surgery. World Journal of Surgery, 35, 1202-1211. https://doi.org/10.1007/s00268-011-1054-2

[5] Felley, C., Perneger, T.V., Goulet, I., Rouillard, C., Azar-Pey, N., Dorta, G., et al. (2008) Combined Written and Oral Information Prior to Gastrointestinal Endoscopy Compared with Oral Information Alone: A Randomized Trial. BMC Gastroenterology, 8, 22. https://doi.org/10.1186/1471-230X-8-22

[6] The National Register of Quality within Gynecological Surgery (GynOp). https://www.gynop.org/omgynop/omgynop.htm

[7] The Swedish Parliament. The Patient Law (2014: 821), Chapter 3-Information. https://www.riksdagen.se/sv/dokument-lagar/dokument/svensk-forfattningssamlin g/patientlag-2014821_sfs-2014-821

[8] Halcomb, E.J., Gholizadeh, L., DiGiacomo, M., Phillips, J. and Davidson, P.M. (2007) Literature Review: Considerations in Undertaking Focus Group Research with Culturally and Linguistically Diverse Groups (Review). Journal of Clinical Nursing, 16, 1000-1011. https://doi.org/10.1111/j.1365-2702.2006.01760.x

[9] Schreier, M. (2012) Qualitative Content Analysis in Practice. Sage Publications, London.

[10] Graneheim, U.H. and Lundman, B. (2004) Qualitative Content Analysis in Nursing Research: Concepts, Procedures and Measures to Achieve Trustworthiness. Nurse Education Today, 24, 105-112. https://doi.org/10.1016/j.nedt.2003.10.001

[11] The Swedish Parliament. The Personal Data Act (2008:355). https://www.riksdagen.se/sv/dokument-lagar/dokument/svensk-forfattningssamlin g/patientdatalag-2008355_sfs-2008-355

[12] The Data Protection Authority. The Personal Data Act. http://www.datainspektionen.se/in-english/legislation/the-personal-data-act/

[13] Herber, O.R., Gies, V., Schwappach, D., Thürmann, P. and Wilm, S. (2014) Patient Information Leaflets: Informing or Frightening? A Focus Group Study Exploring 
Patients' Emotional Reactions and Subsequent Behavior towards Package Leaflets of Commonly Prescribed Medications in Family Practices. BMC Family Practice, 15, 163.

[14] Hopmans, W., Damman, O.C., Timmermans, D.R., Haasbeek, C.J., Slotman, B.J. and Senan, S. (2014) Communicating Cancer Treatment Information Using the Web: Utilizing the Patient's Perspective in Website Development. BMC Medical Informatics and Decision Making, 14, 116. https://doi.org/10.1186/s12911-014-0116-4

[15] Murphy, M., Olivera, C., Wheeler, T. 2nd, Casiano, E., Siddiqui, N., Gala, R., et al. (2013) Postoperative Management and Restrictions for Female Pelvic Surgery: A Systematic Review. International Urogynecology Journal, 24, 185-193. https://doi.org/10.1007/s00192-012-1898-5

[16] Bradford, A. and Meston, C. (2007) Sexual Outcomes and Satisfaction with Hysterectomy: Influence of Patient Education. The Journal of Sexual Medicine, 4, 106-114. https://doi.org/10.1111/j.1743-6109.2006.00384.x

[17] Pakbaz, M., Persson, M., Löfgren, M. and Mogren, I. (2010) “A Hidden Disorder Until the Pieces Fall into Place"-A Qualitative Study of Vaginal Prolapse. BMC Women's Health, 10, 18. https://doi.org/10.1186/1472-6874-10-18

[18] Clarke-Pearson, D.L. and Geller, E.J. (2013) Complications of Hysterectomy. Obstetrics and Gynecology, 121, 654-673. https://doi.org/10.1097/AOG.0b013e3182841594

[19] Wade, J., Pletsch, P.K., Morgan, S.W. and Menting, S.A. (2000) Hysterectomy: What Do Women Need and Want to Know? Journal of Obstetric, Gynecologic and Neonatal Nursing, 29, 33-42. https://doi.org/10.1111/j.1552-6909.2000.tb02754.x

[20] Kain, Z.N., Sevarino, F., Alexander, G.M., Pincus, S. and Mayes, L.C. (2000) Preoperative Anxiety and Postoperative Pain in Women Undergoing Hysterectomy. A Repeated-Measures Design. Journal of Psychosomatic Research, 49, 417-422. https://doi.org/10.1016/S0022-3999(00)00189-6

[21] Spalding, N.J. (2003) Reducing Anxiety by Pre-Operative Education: Make the Future Familiar. Occupational Therapy International, 10, 278-293. https://doi.org/10.1002/oti.191 